\title{
THE SPECTRUM OF NGC 6543 AND ITS NUCLEUS
}

\author{
P. SWINGS*
}

\section{ABSTRACT}

In the nebula NGC 6543 Bowen's fluorescence mechanism is absent, and the recombination process is especially efficient, giving relatively strong lines of $C \mathrm{II}, C \mathrm{III}$, and $N \mathrm{III}$. The nucleus is a Wolf-Rayet star showing lines of $N$ IV and $C$ IV of similar intensities; it is thus an object intermediate between the WN and WC sequences.

I. W. H. Wright ${ }^{\mathrm{I}}$ has shown that the nuclei of planetaries are frequently Wolf-Rayet stars, and he has recorded the wave lengths and intensities of the bright and dark bands in a number of these objects. But heretofore the classification of these Wolf-Rayet spectra in the nitrogen and carbon sequences (or in others) has not been determined with certainty.

On the basis of the data published by Wright, C. S. Beals ${ }^{2}$ has tried to classify the planetary nuclei having spectra of the WolfRayet type, either in the carbon or in the nitrogen sequence. But "since many of these objects are extremely faint and the data consequently meager, too much confidence should not be placed in this list." 3 Beals considers the nucleus of NGC 6543 as a nitrogen star, WN 6. On the other hand, starting from the same data by Wright, C. H. Payne ${ }^{4}$ has classified the nucleus of NGC 6543 among the carbon stars. The laboratory investigations by Edlén have led to a better understanding of the Wolf-Rayet spectra, and his discussion ${ }^{5}$ shows clearly that the spectra of typical Wolf-Rayet stars contain either carbon or nitrogen lines but not both of them with comparable intensities. R. H. Stoy ${ }^{6}$ has obtained spectra of nebulae in the visible region and has expressed the view that the $C$ IV lines $\lambda \lambda{ }_{5800-5812 \text {, }}$ which he observed in the spectrum of NGC 6543, may belong to the nucleus; but no certain decision was reached by him.

2. It is important to reinvestigate the spectra of planetary nuclei

* Contributions from the McDonald Observatory, University of Texas, No. 24.

I Lick Obs. Pub., I3, 2 I I, I918.

${ }_{4} Z_{s . f .} A p .$, 7, I, 1933.

2 Victoria Pub., 4, 286, I930.

5 Zs.f. Ap., 7, 378, г933.

3 Ibid.

${ }^{6}$ Lick.Obs. Bull., I7, I 79, I935. 
of the Wolf-Rayet type. The present paper is concerned with NGC 6543 , which has a sharp nucleus. ${ }^{7}$ Three spectrograms of good definition were obtained with the 82-inch reflector of the McDonald Observatory, two with glass prisms (exposures, I and $2 \frac{1}{2}$ hours; dispersion, $53 \mathrm{~A} / \mathrm{mm}$ at $\lambda$ 4000) and one with quartz prisms (exposure, 2 hours; dispersion, I25 A/mm at $\lambda 4000$ ). In all three cases the emulsion was Agfa Superpan Press. We guided as accurately as possible in order to separate the spectrum of the nucleus from that of the nebula. The spectrograms extend from $\lambda 3400$ to $\lambda 6600$.

3. Lines preseni in the nebular part of the spectrum.-Table I contains all the sharp lines which extend beyond the nucleus. As has already been pointed out by Wright, NGC 6543 shows remarkably clearly the distribution of the physical conditions within the nebula. This appears in a very striking manner on our spectrograms when we compare the shapes of lines belonging to the same atom in different states of ionization, namely, [O I, II, and III]; [S II and III]; $C$ II and $C$ III; and $[N$ II] and $N$ III.

The permitted lines of $N$ III and $C$ iII do not extend far from the nucleus, although their attribution to the nebula is certain because of their sharpness and length. The $C$ II line $\lambda_{426}$ is longer. $^{8}$

4. Excitation processes in the nebula.-For a discussion of the physical conditions prevailing in NGC 6543 it is useful to tabulate the successive ionization potentials of the observed atoms (Table 2).

The absence of $H e$ II 4686 and $O$ III 3760 in the nebula shows that the $N$ III lines are not excited by Bowen's fluorescence mechanism. The observed spectra of $C$ II, $C$ III, and $N$ III are, therefore, all due to recombinations, this process being unusually efficient in this nebula. The third ionization potentials of $C$ and $N$ are almost identical ( $47.64 \mathrm{v}$. and $47.20 \mathrm{v}$.), while the corresponding value for $O$ is appreciably higher $(54.62 \mathrm{v}$.). On the other hand, the ionizing potentials of $O$ III and $H e$ II are practically equal (54.62 v. and 54.I4 v.). The ionization of the nebula by the ultraviolet radiation of the nucleus is such that $\mathrm{C}^{+++}$and $\mathrm{N}^{+++}$are present in the same regions of the nebula which give rise to recombination spectra $(C \mathrm{III}$ and

7 This is the bright planetary in Draco, in which bright lines were discovered by Sir William Huggins.

${ }^{8}$ Wright has attributed the lines of $C \mathrm{II}, C \mathrm{II}$, and $N$ III to the nucleus. 


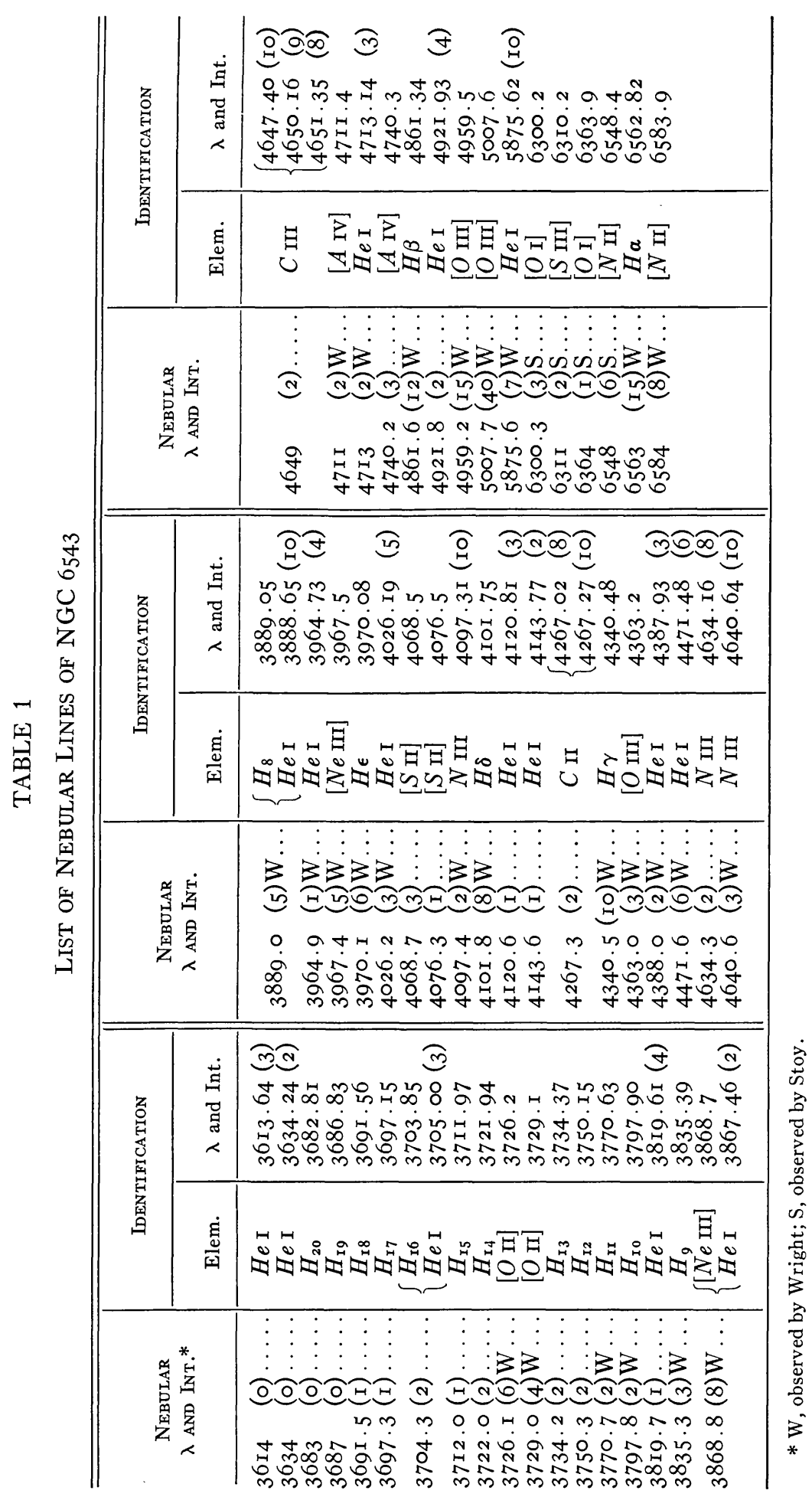


$N$ III), whereas $\mathrm{O}^{+++}$and $H e^{++}$are practically absent. The wave lengths corresponding to $47.5 \mathrm{v}$. and $54.5 \mathrm{v}$. are $\lambda_{260}$ and $\lambda_{228}$, respectively. The observations suggest that the nuclear radiation is much stronger near $\lambda 260$ than near $\lambda 228$ when it reaches the nebular regions. Besides the general decrease of the continuous spectrum toward short wave lengths, this may be due to a crowding of strong, bright, nuclear lines of $H e \mathrm{II}(\lambda \lambda 256 \mathrm{~A}, 243 \mathrm{~A}$, etc.) $O \mathrm{IV}$, $C$ IV, $N$ IV, and $N$ v appearing between $\lambda 260$ and $\lambda$ 230. The region below $\lambda 228$ is much poorer in such lines.

TABLE 2

SucCessive Ionization Potentials of the Atoms OBSERVED IN THE NGC 6543 NEBULA

\begin{tabular}{|c|c|c|c|c|c|c|c|c|}
\hline & $H$ & $\mathrm{He}$ & $C$ & $N$ & $O$ & $\mathrm{Ne}$ & $S$ & $A$ \\
\hline $\begin{array}{l}\text { I. } \\
\text { II. } \\
\text { III. } \\
\text { IV. }\end{array}$ & $\begin{array}{r}\text { I3.53 } \\
\ldots \ldots\end{array}$ & $\begin{array}{l}24 \cdot 46 \\
54 \cdot 14 \\
\ldots \ldots \\
\ldots \ldots\end{array}$ & $\begin{array}{l}\text { II } .20 \\
24.26 \\
47.64 \\
64.17\end{array}$ & $\begin{array}{l}\mathrm{I} 4.46 \\
29.44 \\
47.20 \\
77.04\end{array}$ & $\begin{array}{l}13.55 \\
34.94 \\
54.62 \\
77.03\end{array}$ & $\begin{array}{c}21.47 \\
40.9 \\
63.2 \\
\ldots \ldots\end{array}$ & $\begin{array}{c}10.31 \\
23.3 \\
34.9 \\
\ldots \ldots\end{array}$ & $\begin{array}{c}15.69 \\
27.74 \\
40.7 \\
\ldots \ldots\end{array}$ \\
\hline
\end{tabular}

The simultaneous appearance of $[\mathrm{Ne} \mathrm{III}]$ and $[\mathrm{A} \mathrm{IV}]$ in roughly the same regions is also quite understandable, the ionization potentials of $\mathrm{Ne}^{+}$and $\mathrm{A}^{++}$being $40.9 \mathrm{v}$. and 40.7 v., respectively.

5. Radiations appearing in the nucleus only.-The continuous spectrum of the nucleus is rather strong, and there are not many outstanding bright lines in the astronomical region. Their nuclear character is detected by their width, which is appreciably greater than for the nebular lines, and by their length, which does not extend beyond the continuous spectrum.

Four elements- $\mathrm{He} \mathrm{II}, N \mathrm{IV}, C \mathrm{Iv}$, and $O \mathrm{IV}$ - appear conspicuously in the Wolf-Rayet spectrum; the presence of weak $N$ v and $S i$ IV seems also certain. The comparison is very striking between the $N$ III, $C$ II, or $C$ III nebular lines (long and sharp) and the neighboring $N$ Iv, $C$ iv, or $H e$ II nuclear lines (short and broad).

Helium: $\lambda 4685.8 \mathrm{r}$ of $\mathrm{He} \mathrm{II}$ is a strong nuclear emission (int. 6) with a weak violet absorption; it does not extend beyond the continuous spectrum of the nucleus. 
Nitrogen: The $N$ iv triplet $3 \mathrm{~s}^{3} \mathrm{~S}-3 \mathrm{p}^{3} \mathrm{P}^{0}$ at $\lambda 3478.69$ (lab. int. 7), $\lambda 3482.98$ (5), and $\lambda 3484.90$ (3) is present in emission as a blend of intensity 3 . There is a weak absorption component on the violet side. The $3 \mathrm{P}^{\mathrm{x}} \mathrm{P}^{0}-3 \mathrm{~d}^{\mathrm{x}} \mathrm{D}$ transition at $\lambda 4057.8$ is also present in emission (int. 2) and absorption (int. I).

A faint nuclear line has been measured at $\lambda_{4604}$ and is probably $3 \mathrm{~S}^{2} \mathrm{~S}-3 \mathrm{p}^{2} \mathrm{P}^{0}$ of $N \mathrm{v}(\lambda 4603.2)$.

Carbon: $C \mathrm{IV}$ is identified with certainty by the emission lines listed in Table 3.

TABLE 3

$C$ IV LiNes IN THE NUCLEUS OF NGC 6543

\begin{tabular}{|c|c|c|c|}
\hline$\lambda$ Lab. & $\begin{array}{l}\text { Lab. } \\
\text { Int. }\end{array}$ & Designation & $\begin{array}{c}\text { Int. in } \\
\text { Nucleus }\end{array}$ \\
\hline $\begin{array}{l}4658.54 \ldots \ldots \\
5801.51 \ldots \ldots \\
5812.14 \ldots \ldots\end{array}$ & $\left.\begin{array}{c}5 \mathrm{~d} \\
4 \\
3\end{array}\right\}$ & $\begin{array}{c}5 \mathrm{gf}^{2} \mathrm{G},{ }^{2} \mathrm{~F}-6 \mathrm{hg}^{2} \mathrm{H}^{0},{ }^{2} \mathrm{G}^{0} \\
3 \mathrm{~s}^{2} \mathrm{~S}-3 \mathrm{p}^{2} \mathrm{P}^{0}\end{array}$ & $\left\{\begin{array}{l}3 \\
4 \\
2\end{array}\right.$ \\
\hline
\end{tabular}

The ${ }^{2} \mathrm{~S}-{ }^{2} \mathrm{P}^{\circ}$ doublet was not separated on Wright's plates, but it was on Stoy's spectrograms. The $C$ IV doublet is the most conspicuous feature on our spectrograms of this Wolf-Rayet object, and its attribution to the nucleus is certain by comparison with the nebular lines and also with the nuclear features. The identification of $\lambda_{5} 8 \mathrm{I} 2$ attributed to $C$ IV has been discussed in the ordinary WC stars. ${ }^{9}$ But there is no doubt about the identification in the present case, as we have observed the two components of the doublet with the correct intensity ratio. There is a $N$ IV multiplet $3 \mathrm{p}^{\prime}{ }^{3} \mathrm{P}^{0}-3 \mathrm{~d}^{\prime}{ }^{3} \mathrm{P}$ predicted at $\lambda \lambda_{5} 8 \mathrm{I} 2-5828-5846,{ }^{\text {,o }}$ but its influence on the observed $C$ IV doublet is certainly unimportant.

Oxygen: $O$ III is absent in the nebula, but some weak trace of $\lambda 3759.87$ (strongest laboratory line) may possibly be present in the nucleus.

$O$ IV is characterized by the lines of Table 4 .

Silicon: A trace of $\lambda{ }_{4088.86} S i \mathrm{IV}$ is present.

6. Conclusions concerning the nucleus.-The characteristic lines of the three ions $N \mathrm{IV}, C \mathrm{IV}$, and $O$ IV appear with similar intensities. It

${ }^{9}$ C. S. Beals, Victoria Pub., 6, I29, r934. $\quad$ то Edlén, loc. cit. 
seems that the abundance of carbon is comparable with the abundance of nitrogen or oxygen, although it may be somewhat lower, since the fourth ionization potential is smaller for carbon $(64 . \mathrm{I} 7 \mathrm{v}$.)

TABLE 4

$O$ IV LINES IN THE NUCLEUS OF NGC 6543

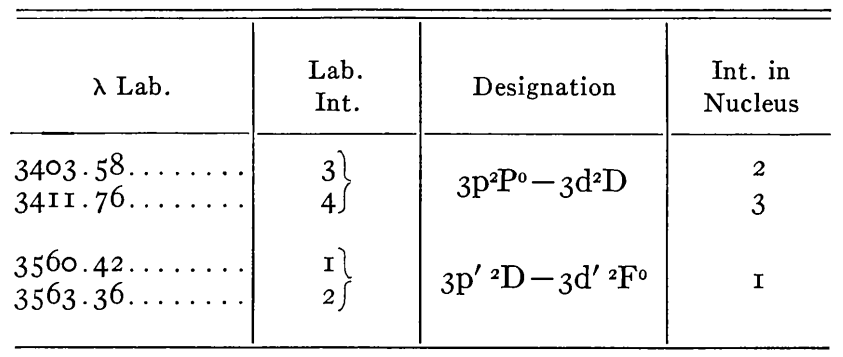

than for nitrogen $(77.04 \mathrm{v}$.) or oxygen $(77.03 \mathrm{v}$.). In any case, as the same effect of the ionization potentials would apply to the usual WN stars, it is safe to assume that the nucleus of NGC 6543 is much richer in carbon than the WN stars and that it is thus an intermediate object between the WN and WC sequences.

MCDONALd OBSERVATORY

March 2I, I940 